\title{
Membership Roster
}

This Roster is a list of members and subscribers as of April 1, 1957. An asterik preceding the name means sustaining member. Members and subscribers who have not paid the dues for 1957 are urgently requested to do so immediately in order to insure the uninterrupted delivery of Names.

I.

Charles C. Abbott, Grad. School of Bus. Admin., Univ. of Virginia, Charlottesville, Va.

Samuel H. Abramson, 294 Fisher Ave., White Plains, N.Y.

*Henry E. Abt, Brand Names Foundation, 437 Fifth Ave., New York 16, N.Y.

Louise M. Ackerman, 333 W Calvert St., Lincoln, Nebr.

K. T. Adams, 5110 Mesa Terrace, La Mesa, Calif.

Curtis Adler, 10978 Bluffside Dr., North Hollywood, Calif.

Air University Library, Maxwell A. F. B., Ala.

University of Alabama, University, Ala.

James E. Alatis, 224 W First Ave., Columbus 1, Ohio

Harold B. Allen, 317 Folwell Hall, Univ. of Minnesota, Minneapolis 14, Minn.

Morse S. Allen, 3 Fern St., Hartford 5, Conn.

Karl Ames, 383 Oak Ave., River Edge, N.J.

American Antiquarian Society, Park and Salisbury, Worchester 5, Mass. American Geographical Society, Broadway at 156th St., New York 32, N.Y.

American Peoples Encyclopaedia, 179 N Michigan Ave., Chicago 15, Ill. Amerikanische Gedenkbibliothek, Berlin SW. 61, Germany

Arizona State College, Tempe, Ariz.

University of Arizona, Tucson, Ariz.

University of Arkansas, Fayetteville, Ark.

*William E. Ashton, 712 Fifth Ave., Helena, Mont.

Mrs. F. O. Atha, 9 High St., Mannington, W. Va.

Atlanta Public Library, 126 Carnegie Way N. W., Atlanta 3, Ga.

E. Bagby Atwood, Dept. of English, Univ. of Texas, Austin, Tex.

Donald Gay Baker, 8th Ave., Collegeville, Pa.

*Clarence L. Barnhart, 141 Parkway Road, Bronxville 8, N.Y.

Robert K. Barnhart, 3 Tanglewylde Ave., Bronxville, N.Y.

S. V. Baum, English Dept., Temple Univ., Philadelphia 22, Pa.

Baylor University, Box 307B, Univ. Sta., Waco, Texas

Richard Beck, Dept. of Languages, Univ. of North Dakota, Grand Forks, N. Dak. 
Madison S. Beeler, 2413 Dwinelle Hall, Univ. of California, Berkeley 4, Calif.

Allen Belden, 7055 Wyndale St., N. W., Washington 15, D.C.

Handsel G. Bell, 1605 Fairview St., Berkeley 3, Calif.

A. I. Bereskin, Controller of Surveys, Dept. of Natl. Resources, Regina, Sask., Canada

George Bijur, Hotel Lombardy, 111 E. 56th St., New York 22, N.Y.

Bodleian Library, Oxford, England

A. Boodberg, 105 Durant Hall, Univ. of California, Berkeley 4, Calif.

Boston Public Library, History Dept., Copley Sq., Boston 17, Mass.

Bowdoin College Library, Brunswick, Maine

Bowling Green State University, Bowling Green, Ohio

*F. W. Bradley, 4250 St. Clair Dr., Columbia 4, S.C.

Bradley University Library, Peoria, Ill.

E. L. Brainerd, 2814 Polk St., N. E., Minneapolis 18, Minn.

Felix Brecher, $450 \mathrm{~W} 162$ nd Street, New York 32, N.Y.

A. A. Brierly, Independence, Calif.

Brigham Young University, Provo, Utah

University of British Columbia, Vancouver 8, B. C., Canada

Brooklyn Public Library, Grand Army Plaza, Brooklyn 38, N.Y.

Samuel L. Brown, 2522 N. E. 4th St., Minneapolis 18, Minn.

Thomas P. Brown, 615 Alvarado St., San Francisco 14, Calif.

Brown University Library, Providence 12, R.I.

Arthur W. Browne, 623 Palo Alto Ave., Mountain View, Calif.

Office International de Librairie, 184 Rue de l'Hôtel des Monnaies,

Brussels, Belgium

Edwin H. Bryan, Jr., B. P. Bishop Museum, Honolulu 17, Hawaii

Margaret M. Bryant, 1 Montague Ter., Brooklyn 2, N.Y.

Bryn Mawr College, Bryn Mawr, Pa.

Bucknell University, Ellen Clarke Bertrand Library, Lewisburg, Pa.

Warren H. Buell, 608 N Cherokee Ave., Los Angeles 4, Calif.

University of Buffalo, Lockwood Memorial Library, 3435 Main St., Buffalo 14, N.Y.

Meredith F. Burrill, U. S. Bd. on Geogr. Names, Washington 25, D.C.

Mrs. A. Kyle Bush, 5 Bush Ave., Philippi, W.Va.

Robert A. Caldwell, Dept. of English, Univ. of North Dakota, Grand Forks, N.Dak.

California Academy of Sciences, Golden Gate Park, San Francisco 18, Calif.

University of California Library, Berkeley 4, Calif.

Dept. of Geography, Univ. of California, Berkeley 4, Calif.

University of California Library, Davis, Calif.

University of California Library, Los Angeles 24, Calif. 


\section{Membership Roster}

University of California, Riverside, Calif.

California State Library, Sacramento 14, Calif.

Arthur L. Campbell, 5066 Romaini St., Los Angeles 29, Calif.

Henry C. Carlisle, 315 Montgomery St., San Francisco, Calif.

Helen Carlson, Box 246, Univ. of New Mexico, Albuquerque, N. Mex.

A. Carnoy, Louvain, Belgium

Frederic G. Cassidy, 315 Bascom Hall, Madison 6, Wisc.

Ava H. Chadbourne, 140 Hammond St., Bangor, Maine

Ben F. Cheek, Jr., P. O. Box 387, Toccoa, Ga.

Chicago Public Library, 78 E.Washington, Chicago 2, Ill.

University of Chicago, Periodic. Dept., Harper M22, Chicago 37, Ill.

Lewis H. Chrisman, 87 Smithfield St., Buckhannon, W.Va.

Public Library of Cincinnati, 629 Vine St., Cincinnati 2, Ohio

Marjorie Clagett, Western State College, Bowling Green, Ky.

Donald T. Clark, 25 Moon Hill Rd., Lexington, Mass.

Dwight L. Clarke, 434 Muirfield Rd., Los Angeles 5, Calif.

Public Library, 325 Superior Ave., Cleveland 14, Ohio

R. T. Cochran, 1047 Simmons St., Kirkwood 22, Mo.

D. B. Cofer, Box 211, Faculty Exchange, College Sta., Texas

Hennig Cohen, Exec. Secy., American Studies Assn., Univ. of Pennsylvania, Philadelphia $4, \mathrm{~Pa}$.

Charles Collins, 716 Brummel St., Evanston, Ill.

Henry E. Collins, 117 N Lotus Ave., Chicago 44, Ill.

Colorado College Library, Colorado Springs, Colo.

University of Colorado Libraries, Boulder, Colo.

Connecticut State Library, Hartford, Conn.

Franklin D. Cooley, Univ. of Maryland, College Park, Md.

Joseph N. Corcoran, 1304 Phila. Natl. Bk. Bldg., Philadelphia 7, Pa.

Cornell University Library, Ithaca, N.Y.

E. H. Criswell, Univ. of Tulsa, Tulsa 4, Okla.

Ephraim Cross, 215 W. 98 St., New York 25, N.Y.

Thomas L. Crowell, 15 Claremont Ave., New York 27, N.Y.

Jack A. Dabbs, 1011 Edgewood, Bryan, Texas

Dallas Public Library, 1926 Commerce St., Dallas 1, Texas

Orlando D'Amato, 394 Highland Ave., Orange, N.J.

Dartmouth College, Baker Library, Hanover, N.H.

Levette J. Davidson, Univ. of Denver, Denver 10, Colo.

Moshe Davis, Jewish Theolog. Seminary, Broadway and 122nd St., New

York 27, N.Y.

Orpha M. Dean, 1241/2 Sibley St., Hammond, Ind.

Robert N. DeArmond, Box 1571, Juneau, Alaska

Demarest Memorial Library, 637 Kinderkamack Rd., Oradell, N.J.

Denver Public Library, Civic Center, Denver 2, Colo. 
University of Denver Library, Denver, Colo.

Detroit Public Library, 5201 Woodward Ave., Detroit 2, Mich.

University of Detroit Library, 4001 W McNichols Rd., Detroit 21, Mich.

Geart Droege, 5112 Broadway, Chicago 40, Ill.

Audrey R. Duckert, 6 Ash St., Cambridge 38, Mass.

Duke University Library, Durham, N.C.

R. M. Duncan, Univ. of New Mexico, Albuquerque, N.M.

A. R. Dunlap, 302 Hullihen Hall, Newark, Del.

Gilles Dupré, 10145 D'Auteuil, Montreal 12, P. Q., Canada

S. J. Eckerson, General Drafting Co., Convent Sta., N.J.

Edith F. Edward, 7118 East End Ave., Chicago 49, Ill.

Edward C. Ehrensperger, Univ. of South Dakota, Vermillion, S.Dak.

Enoch Pratt Free Library, 400 Cathedral St., Baltimore 1, Md.

Mrs. Willis J. Erlandson, 401 Prospect St., Lake Mills, Wisc.

The Erskine College Library, Due West, S.C.

*Francis P. Farquhar, 2930 Avalon Ave., Berkeley 5, Calif.

Thomas P. Field, Univ. of Kentucky, Lexington, Ky.

Nell Green Findley, Box 217, Canyon, Texas

Florida State Library, Supreme Court Bldg., Tallahassee, Fla.

Florida State University Libraries, East Campus, Tallahassee, Fla.

University of Florida Libraries, Gainesville, Fla.

*C. S. Forester, 1066 Park Hills Rd., Berkeley, Calif.

Theodore G. Foster, 2426 Strathmore Rd., Lansing 10, Mich.

Gösta Franzen, Faculty Exchange, Univ. of Chicago, Chicago 37, Ill.

Freie Universität, Berlin-Dahlem, Garystr. 45, Germany

Fresno State College Library, Fresno, Calif.

Joseph G. Fucilla, Dept. of Romance Languages, Northwestern University, Evanston, Ill.

Funk \& Wagnalls Company, Edit. Ref. Library, 153 E. 24th St., New York 10, N.Y.

*J. C. Furnas, R. D. 1, Lebanon, N.J.

Wilbur G. Gaffney, 121 Andrews Hall, Univ. of Nebraska, Lincoln 8, Nebr. Gaston County Public Library, Gastonia, N.C.

James A. Geary, 4306 - 28 th Place, Mt. Rainier, Md.

Mrs. Davis E. Geiger, 509 Amanda Furnace Dr., Ashland, Ky.

I. J. Gelb, Univ. of Chicago, Chicago, Ill.

Demetrius J. Georgacas, Univ. of North Dakota, Grand Forks, N.Dak.

William H. Gilbert, 9015 First Ave., Silver Springs, Md.

Charles Edgar Gilliam, 220 Union Trust Bldg., Petersburg, Va.

Mary E. Griffin, Vassar College, Poughkeepsie, N.Y.

C. M. Goethe, Capital Natl. Bank Bldg., 7 th and J. Streets, Sacramento 14, Calif.

Niedersächs. u.Universitätsbibliothek, Prinzenstr. 1, Göttingen, Germany 


\section{Membership Roster}

*Ben Grauer, 29 E. 63rd St., New York 21, N.Y.

Ewald T. Grether, School of Bus. Admin., Univ. of Calif., Berkeley 4, Calif.

Grosvenor Library, 383 Franklin St., Buffalo 2, N.Y.

Elisabeth K. Gudde, P. O. Box 567, Orinda, Calif.

*Erwin G. Gudde, P. O. Box 567, Orinda, Calif.

Gumperts Aktie Bolag, Goeteborg, Sweden

P. Haase \& Son, Levstrede 8, Copenhagen K, Denmark

Richard N. Hall, 5914 Wilmette Rd., Bethesda 14, Md.

C. H. Handschin, 405 Vine St., Oxford, Ohio

John Louis Haney, 6419 Woodpine Ave., Philadelphia 31, Pa.

Louise Hanley, 7141/2 - 3rd St., Wausau, Wisc.

Kelsie B. Harder, Dept. of Languages and Literature, Youngstown College, Youngstown, Ohio

Lester Hargrett, 2106 Spence St., Tallahassee, Fla.

John P. Harrington, 125 W. Carrillo St., Santa Barbara, Calif.

Jesse W. Harris, Southern Illinois Univ., Carbondale, Ill.

*James D. Hart, 740 San Luis Rd., Berkeley 7, Calif.

A. A. Hartman, Rm. 1004, 1121 S. State St., Chicago 5, Ill.

Harvard College Library, Cambridge 38, Mass.

Lee F. Hawley, 1 Langdon St., Madison 3, Wisc.

David Judson Haykin, Library of Congress, Washington 25, D.C.

Frederick W. Hilbig, 1149 Elgin Ave., Salt Lake City 1, Utah

Lewis Heck, 3421 Northampton St., Washington 15, D.C.

Atcheson L. Hench, Univ. of Virginia, Charlottesville, Va.

*Robert Hitchman, 611-13 th Ave. N., Seattle 2, Wash.

Cecilia A. Hotchner, Hunter College, 695 Park Ave., New York 21, N.Y.

Henry Beetle Hough, Edgartown, Mass.

Arthur H. Hughes, Trinity College, Hartford 6, Conn.

Humanistiska Biblioteket, Stockholms Högskola, Stockholm 6, Sweden Ocie Hunt, 105 W. 3rd St., Sweetwater, Texas

Henry E. Huntington Library, San Marino, Calif.

Indiana State Library, 140 N. Senate Ave., Indianapolis 4, Ind.

University of Illinois Library, Urbana, Ill.

State Historical Society of Iowa, Iowa City, Iowa

State University of Iowa, Iowa City, Iowa

Walter V. Irving, 3 Mather St., Binghamton, N.Y.

Helen P. Jackson, Californian Hotel, San Francisco 2, Calif.

John B. Jackson, Box 2149, Santa Fe, N.M.

Moritz Jagendorf, 260 Riverside Dr., New York, N.Y.

Assar Janzén, Univ. of California, Berkeley 4, Calif.

Edgar Jenkins, Box 607, Palm Desert, Calif.

Philip N. Jenner, DAC, 500th MIG, Transl., APO 613, c/o Postmaster, San Francisco, Calif. 
Kiichi Jinbo, 8758 Bebaseki, Fuchu-shi, Tokyo, Japan

Public Library, Market Sq., Johannesburg, South Africa

John Carroll University, University Heights, Cleveland 18, Ohio

Johns Hopkins University Library, Baltimore 15, Md.

Joint University Libraries, Kirkland Hall, Nashville, Tenn.

E. Gustav Johnson, 3225 Foster Ave., Chicago 25, Ill.

- Petras Jonikas, 281 Olmstead Rd., Riverside, Ill.

Rabbi Benzion C. Kaganoff, 2708 W. Greenleaf, Chicago 45, Ill. .

Public Library, Kansas City 6, Mo.

Fort Hays Kansas State College Library, Hays, Kansas

University of Kansas Library, Lawrence, Kansas

Henry Karpenstein, 80 Ord St., San Francisco 17, Calif.

Allen B. Kellogg, 1411 Loretta Dr., Indianapolis 27, Ind.

Hamill Kenny, P. O. Box 681, Franconia, N.H.

Kent State University, Kent, Ohio

University of Kentucky Libraries, Lexington, Ky.

John S. Kenyon, Hiram College, Hiram, Ohio

Joyce King, 40 Swains Lane, Highgate, London, N. 6, England

Clark Kinnaird, 76 Mine St., Flemington, N.J.

Helge Kökeritz, Yale Univ., New Haven, Conn.

Panteleimon K. Kovaliv, 518 - 9 th St., N. E., Apt. 410, Washington 2, D.C.

Walter C. Kraft, Dept. of Mod. Lang., Oregon State College, Corvallis, Ore.

Hans Krahe, Indogerm. Seminar, Universität, Tübingen, Germany

Fritz L. Kramer, Mackay School of Mines, Univ. of Nevada, Reno, Nev.

Kungl. Biblioteket, Stockholm, Sweden

Edward Kupka, 1391 Rose St., Berkeley 2, Calif.

Aneale L. Kushmar, 2735 Taylor, Detroit 6, Mich.

Cahrilaos G. Lagoudakis, 3606 Kanawaha St., N. W., Washington, D.C.

Symington P. Landreth, 1 Pine Grove, Bristol, Pa.

Hector Lee, Chico State College, Chico, Calif.

Lehigh University Library, Bethlehem, Pa.

*John Leighly, 1435 Arch St., Berkeley 8, Calif.

Agnes C. Leland, 47 Avis Rd., Berkeley 7, Calif.

Bibliotheka Akademii Nauk SSSR, Birgevaia 1, Leningrad 164, U.S.S.R.

H. K. Lewis \& Co., Ltd., P. O. Box 66, 136 Gower St., London W. C. 1, England

Lincoln Library, Shippensburg, Pa.

Edwin M. Loeb, 1565 La Vereda Rd., Berkeley 8, Calif.

*Grant Loomis, 828 Coventry Rd., Berkeley 7, Calif.

Los Angeles Public Library, 630 W., 5th St., Los Angeles 17, Calif.

Los Angeles State College, 855 N. Vermont St., Los Angeles 29, Calif. 
Louisiana State University Library, Baton Rouge 3, La. Public Library, 301-333 Library Place, Louisville 3, Ky. Bibliothèque de l'Université, Pl. Mgr. Ladeuze, Louvain, Belgium Hobart M. Lovett, 1177 Cragmont Ave., Berkeley 8, Calif.

A. B. Lundequistska Bokhandeln, Uppsala, Sweden John Lyman, 7801 Gateway Blvd., Washington 28, D.C. W. L. McAtee, 2 Davie Circle, Chapel Hill, N. C. Eugene S. McCartney, 202 Michigan Union, Ann Arbor, Mich. A. R. McCrady, 1030 E. Green St., Pasadena 1, Calif. John T. McCutcheon, Jr., Chicago Tribune, Chicago 11, Ill. John Francis McDermott, 6345 Westminster Place, St. Louis 5, Mo. ${ }^{*}$ Gordon McKenzie, Dept. of English, Univ. of Calif., Berkeley 4, Calif. James B. McMillan, Drawer 2877, University, Ala.

E. Wallace McMullen, 57 E. Circle Dr., East Longmeadow, Mass. Alexander McQueen, $5222 \mathrm{~N}$. Lakewood Ave., Chicago 40, Ill. James M. McWilliams, 697 Arlington Ave., Berkeley 7, Calif. Ernest Maas, 150-76 Village Rd., Parkway Village, Jamaica 32, N.Y. James G. W. MacClamroch, Jefferson Standard Bldg., Greensboro, N.C. Elsie Mag, G. \& C. Merriam Co., 47 Federal St., Springfield 2, Mass. Yakov Malkiel, 1 Arlington Lane, Berkeley 7, Calif.

*Kemp Malone, Dept. of English, Johns Hopkins Univ., Baltimore 18, Md.

Frederick Manfred, 6717 Auto Club Rd., Minneapolis 20, Minn.

University of Manitoba Library, Winnipeg, Manit., Canada

M. R. Manolatos, 5025 W. Monroe St., Chicago 44, Ill.

Albert H. Marckwardt, Univ. of Michigan, Ann Arbor, Mich.

Max Markrich, 248 - 15th Ave., San Francisco 18, Calif.

University of Maryland Library, College Park, Md.

Mamie Meredith, 2340 Sumner St., Lincoln 2, Nebr.

Louis Mesko, Valley Forge Rd., Devon, Pa.

*Theodore R. Meyer, 3528 Washington St., San Francisco 18, Calif.

Miami Public Library, I Biscayne Blvd., Miami 32, Fla.

Miami University Library, Oxford, Ohio

A. F. Michaelis, Standard Oil Co., 225 Bush St., Rm. 2174, San Francisco, Calif.

Michigan Historical Commission, Lansing, Mich.

Michigan State College, East Lansing, Mich.

Michigan State Library, Lansing, Mich.

University of Michigan Library, Ann Arbor, Mich.

Milwaukee Public Library, 814 W. Wisconsin Ave., Milwaukee 3, Wisc. Minneapolis Public Library, 10th and Hennepin, Minneapolis 3, Minn. University of Minnesota Library, Minneapolis 14, Minn.

Arthur Minton, R. F. D. 3, New Milford, Conn. 
Missouri State Library, State Office Bldg., Jefferson City, Mo.

University of Missouri Library, Columbia, Mo.

William E. Mockler, Dept. of English, West Virginia Univ., Morgantown, W.Va.

Historical Society of Montana, Helena, Mont.

S. G. Morley, 2635 Etna St., Berkeley 4, Calif.

Maralyn Morton, Idaho State College, Pocatello, Idaho

Bibliotheka Akademii Nauk SSSR, Ul Frunze 11, Moscow 19, U.S.S.R.

Universitätsbibliothek, Geschwister Scholl Pl. 1, 13B München 22, Germany

G. M. Munroe, Secretary, Bd. on Geographical Names, No. 8 Temporary Bldg., Ottawa, Ont., Canada

John G. Mutziger, 4711 Riverdale Rd., Riverdale, Md.

National Audubon Society Library, 1130 Fifth Ave., New York 25, N.Y.

National Geographic Society, 16 th and M Streets N. W., Washington 6, D.C.

Nebraska State Historical Society, 1500 R St., Lincoln 8, Nebr.

Claude Henry Neuffer, Univ. of South Carolina, Columbia 1, S.C.

University of Nevada Library, Reno, Nevada

Newark Public Library, 5 Washington St., Newark 1, N.J.

The Newberry Library, Chicago 10, Ill.

New Haven Public Library, New Haven 11, Conn.

University of New Mexico Library, Albuquerque, N.M.

New York Historical Society, 170 Central Park West, New York 24, N.Y.

New York Public Library, 5th Ave. and 42nd St., New York 18, N.Y.

New York University, Washington Square Library, 100 Washington

Square East, New York 3, N.Y.

Hidemasa Nishizawa, 1-1 Nagato-Cho, Chiyoda-Ku, Tokyo, Japan

Public Library, 345 W. Freemason St., Norfolk 10, Va.

Delf Norona, 315 Seventh St., Moundsville, W.Va.

University of North Carolina Library, Chapel Hill, N.C.

Women's College, Univ. of North Carolina, Greensboro, N.C.

University of North Dakota, University Station, Grand Forks, N. Dak. Northern Illinois State Teachers College, Dekalb, Ill.

Northwestern University Library, Evanston, Ill.

University of Notre Dame, Notre Dame, Ind.

Benjamin Nunez, Earl Hall, Columbia Univ., New York, N.Y.

Public Library, 125 - 14th St., Oakland, Calif.

Clarence B. Odell, Denoyer-Geppert Co., 5235 Ravenswood Ave., Chicago 40 , Ill.

Ohio State University Library, Columbus 10, Ohio

Oklahoma A. and M. College Library, Stillwater, Okla.

Oklahoma City Libraries, 3rd and Robinson, Oklahoma City 2, Okla. 
University of Oklahoma, Norman, Okla.

University of Oregon Library, Eugene, Oregon

Allan H. Orrick, Indiana Univ., Bloomington, Ind.

National Museum od Canada, Library, Ottawa 4, Ont., Canada

Librarian, Geographical Branch, 100 Elgin Bldg., Ottawa 4, Canada

Wm. D. Overman, 31 Mull Ave., Akron 3, Ohio

Bibliothèque de l'Université, 47 Rue des Ecoles, Paris 5, France

Librairie des Méridiens, Klincksieck \& Cie., 119 Blvd. St. Germain,

Paris VI, France

Pennsylvania State Library, Box 608, Harrisburg, Pa.

Patee Library, Pennsylvania State Univ., University Park, Pa.

John P. Pauls, 3209 W. Evergreen Ave., Chicago 51, Ill.

Peabody Museum Library, Salem, Mass.

T. M. Pearce, Univ. of New Mexico, Albuquerque, N.M.

Historical Society of Pennsylvania, 130 Locust St., Philadelphia 7, Pa.

University of Pennsylvania Library, 34th St., and Woodland Ave., Philadelphia 4, Pa.

Alfred Percy, Elon Rd., Madison Heights, Va.

P. G. Perrin, Department of English, University of Washington, Seattle, Wash.

Benedict Pfaller, OSB, St. Anthony's Church, St. Anthony, N.D.

Charles A. Philhower, 303 Mountain Ave., Westfield, N.J.

University of Pittsburgh Library, Pittsburgh 13, Pa.

George H. Pollock, Institute for Psychoanalysis, $664 \mathrm{~N}$ Michigan Ave, Chicago 11.

Library Assoc. of Portland, 801 S. W. 10th Ave., Portland 5, Oregon Louise Pound, 1632 L Street, Lincoln 8, Nebr.

Pratt Institute Library, Brooklyn 5, N.Y.

Newbell N. Puckett, Dept. of Sociology, Western Reserve Univ., Cleveland 6, Ohio

University of Puerto Rico Library, Rio Piedras, Puerto Rico

Mrs. H. Pugh, 130 W. Broad St., Burlington, N.J.

Ernst Pulgram, Dept. of Romance Languages, Univ. of Michigan, Ann Arbor, Mich.

State College Library, Pullman, Wash.

Purdue University Library, Lafayette, Ind.

Thomas Pyles, Anderson Hall, Univ. of Florida, Gainesville, Fla.

Queens Borough Public Library, Jamaica, N.Y.

Queens University Library, Kingston, Ont., Canada

Manuel L. Quezon Institute, P. O. Box 2931, Manila, Philippines

C. J. Raeth, Upham Hall, Miami University, Oxford, Ohio

Rand McNally \& Co., P. O. Box 7600, Chicago 80, Ill.

H. F. Raup, Dept. of Geog. and Geol., Kent State Univ., Kent, Ohio 
Allen Walker Read, 39 Claremont Ave., New York 27, N.Y.

Click Relander, 1212 N. 32nd Ave., Yakima, Wash.

George Rentz, Box 1437, Aramco, Dhahran, Saudi Arabia

T. W. Reynolds, Box 121, Riviera Beach, Fla.

The Rice Institute, Fondren Library, Box 1892, Houston 1, Texas

Richmond Public Library, 101 E. Franklin St., Richmond 19, Va.

John S. Roberts, 469 - 3rd St. W., Sonoma, Calif.

R. D. Roberts, 312 Shearer Bldg., Bay City, Mich.

Public Library, 115 South Ave., Rochester, N.Y.

P. Burwell Rogers, English Dept., Bucknell Univ., Lewisburg, Pa.

Morris Rosenblum, 959 Carroll St., Brooklyn 25, N.Y.

Stowell Rounds, Cannondale, Conn.

Hubert A. Royster, 2318 Beechridge Rd., Raleigh, N.C.

J. B. Rudnyckyj, Dept. of Slavic Studies, Univ. of Manitoba, Winnipeg, Manit., Canada

St. Louis Public Library, 13th and 14th Sts., St. Louis 3, Mo.

Biblioteca de la Facultad de Filosofia, Plaza de la Anaya, Salamanca, Spain

Wheeler Sammons, Jr., A. N. Marquis Co., 210 E. Ohio St., Chicago 11, Ill.

San Diego Public Library, San Diego 1, Calif.

Public Library, Civic Center, San Francisco 2, Calif.

San Jose Junior College, 2100 Moorpark Ave., San Jose, Calif.

San Jose State College Library, San Jose, Calif.

William Saroyan, 24848 Malibu Rd., Malibu, Calif.

Margaret C. Schemel, Lincoln Memorial Univ., Harrogate, Tenn.

Richard B. Sealock, 5819 McGee St., Kansas City 13, Mo.

William M. Seaman, Dept. of Foreign Lang., Michigan State College, East Lansing, Mich.

Seattle Public Library, Seattle 4, Wash.

Pauline A. Seely, 1135 Grant St., Denver 3, Colo.

Alfred Senn, 207 Cynwyd Rd., Bala-Cynwyd, Pa.

George Pope Shannon, 4522 Drummond Ave., Chevy Chase 15, Md.

Esther K. Sheldon, 352 Argyle Rd., Brooklyn 18, N.Y.

James Sledd, Dept. of English, Univ. of Calif., Berkeley 4, Calif.

Elsdon C. Smith, 322 Sherman Ave., Evanston, Ill.

Smithsonian Institution, Library, Washington 25, D.C.

Robert Sonkin, City College, 139 th St. and Convent Ave., New York 31, N.Y.

University of South Carolina, Library, Columbia 1, S.C.

University of South Dakota Libraries, Vermillion, S.D.

Southern Illinois University, Carbondale, Ill.

Southwest Museum Library, Highland Park, Los Angeles 42, Calif.

Southwestern Louisiana Inst., Stephens Memorial Library, Lafayette, La. 


\section{2}

David G. Speer, Dept. of Modern Lang., Purdue Univ., Lafayette, Ind. Spokane Public Library, Spokane 4, Wash.

*Gustavo Stahl, 10a Calle 6-13, Z 1, Guatemala City, Guatemala Stanford University Libraries, Stanford, Calif.

Taylor Starck, Harvard Univ., Cambridge 38, Mass.

D. T. Starnes, Univ. of Texas, Austin 12, Texas

*W. A. Starr, 405 Montgomery St., San Francisco 4, Calif.

Vilhjalmur Stefansson, Dartmouth College, Baker Library, Hanover, N.H. Jess Stein, Random House, 457 Madison Ave., New York 22, N.Y.

Mrs. Mary Florence Steiner, 225 Central Park Ave., Wilmette, Ill.

Noel C. Stevenson, 926 J Bldg., Suite 612, Sacramento 14, Calif.

Mrs. Andrew W. Stewart, 1591 Oakdale St., Pasadena, Calif.

*George R. Stewart, 100 Codornices Rd., Berkeley, Calif.

Sterling A. Stoudemire, Dept. of Romance Languages, Univ. of North Carolina, Chapel Hill, N.C.

O. Paul Straubinger, 4607 Oakwood P1., Riverside, Calif.

Arval L. Streadbeck, A 256 University of Utah, Salt Lake City 1, Utah E. G. Swem, 119 Chandler Court, Williamsburg, Va.

Tacoma Public Library, Tacoma 3, Wash.

Ashley F. Talbot, 38 Winans St., East Orange, N.J.

*Peter Tamony, 2876 - 24th St., San Francisco 10, Calif.

Edward Taube, Univ. of Wisconsin, 620 Lake Ave., Racine, Wisc.

Tennessee State Library and Archives, Nashville 3, Tenn.

University of Tennessee Library, Knoxville 16, Tenn.

I. J. Tepperman, 548 Palmerston Blvd., Toronto 4, Ont., Canada

A. and M. College of Texas, College Station, Texas

Texas Christian University Library, Fort Worth 9, Texas

University of Texas Library, Austin 12, Texas

Texas State College for Women, Box 3715, Denton, Texas

Texas Technological College Library, Lubbock, Texas

Gutierre Tibn, Paseo de la Reforma 1-959, Ciudad de Mexico, Mexico

Toledo Public Library, 325 Michigan St., Toledo 2, Ohio

University of Toledo Library, Toledo, Ohio

University of Tulsa Library, 7 th and College, Tulsa, Okla.

Harlan L. Umansky, Emerson High School, Union City, N.J.

U.S. Army Map Service, 6500 Brooks Lane, Washington 16, D.C.

U.S. Coast and Geodetic Survey, Library, Washington 25, D.C.

U.S. Dept. of the Interior, Office of Geography, Rm. 2463, Interior Bldg., Washington 25, D.C.

U.S. Geological Survey Library, Rm. 1033, GSA Bldg., Washington 25, D.C. U.S. Navy Hydrographic Office, Suitland, Md.

The Genealogical Society, 80 N. Main St., Salt Lake City 1, Utah

Utah State Agricultural College, Logan, Utah 
University of Utah, Salt Lake City, Utah

Francis L. Utley, Dept. of English, Ohio State Univ., Columbus 14, Ohio

Michael S. Vartanoff, 6825 Wilson Lane, Bethesda 14, Md.

Vermont Historical Society, Montpelier, Vt.

Eugene B. Vest, Chicago Undergrad. Div., Univ. of Illinois, Chicago 11,Ill.

James M. Vicary, 22 East 60 th Street, New York 22, N.Y.

Provincial Library, Victoria, B. C., Canada

Virginia Historical Society, The Lee House, 707 E. Franklin St., Richmond 19, Va.

Virginia State Library, Richmond 19, Va.

Ivan H. Walton, 320 Lakeview Dr., Ann Arbor, Mich.

University of Washington Library, Seattle 5, Wash.

Frank H. Waskey, Oakville, Wash.

R. Gordon Wasson, 23 Wall St., New York 8, N.Y.

Wayne State University Library, 5210 - 2nd Ave., Detroit 2, Mich.

C. A. Weslager, $601 \mathrm{~S}$. Maryland Ave., Wilmington, Del.

West Virginia University Library, Morgantown, W.Va.

West Virginia Wesleyan College Library, Buckhannon, W.Va.

Western Reserve University Library, 11111 Euclid Ave., Cleveland 6, Ohio

H. J. Van de Wijer, E. Van Evenstraat 10, Louvain, Belgium

Helen P. Wheeler, Vassar College, Poughkeepsie, N.Y.

Catherine Poey Williams, 603 Ocean Ave., Santa Monica, Calif.

Margery M. Williams, 63 Whittredge Rd., Summit, N.J.

A. H. Wilson, 28 Warren Ave., Somerville 43, Mass.

John N. Winburne, 1400 N. Hagadorn Rd., East Lansing, Mich.

University of Wisconsin Library, Madison 6, Wisc.

Wisconsin State Historical Society, 816 State St., Madison 6, Wisc.

University of the Witwatersrand Library, Milner Park, Johannesburg, South Africa

Hubert Blaine Wolfeschlegelsteinhausenbergerdorff, Sr., 142 South 11 th St., Philadelphia 7, Pa.

H. J. Woods, Jr., 511 Fairview Ave., Reno, Nev.

Henry B. Woolf, 151 Maple St., Springfield, Mass.

The World Publishing Co., David B. Guralnik, 2231 W. 110 St., Cleveland 2, Ohio

A. J. Wraight, 18 - 9 th St., N. E., Washington 2, D.C.

Charles L. Wrenn, Pembroke College, Oxford, England

University of Wyoming, Laramie, Wyo.

Yale University Library, New Haven, Conn.

*Walter Yust, Editor, Encyclopaedia Brittanica, 425 N. Michigan Ave., Chicago 11, Ill.

Wilbur Zelinsky, 20232 San Juan Dr., Detroit 21, Mich.

Gertrude M. Zimmer, 221 Patterson St., Farmington, Mo. 


\section{II.}

In the following section, members are listed according to the state or foreign country in which they reside. Of the numbers in parentheses, the first refers to personal members, the second to libraries.

Alabama (1-2). McMillan, James B.; Air University Library - University of Alabama

Arizona (0-2). Arizona State College - University of Arizona

Arkansas (0-1). University of Arkansas

California (49-18). Adams, K. T. - Beeler, Madison S. - Bell, Handsel G. - Boodberg, P. A. - Brierly, A. A. - Brown, Thomas P. - Browne, Arthur W. - Buell, Warren H. - Campbell, Arthur L. - Carlisle, Henry C. - Clarke, Dwight L. - Farquhar, Francis P. - Forester, G. S. Goethe, C. M. - Grether, Ewald T. - Gudde, Elisabeth K. - Gudde, Erwin G. - Harrington, John P. - Hart, James D. - Jackson, Helen P. - Janzén, Assar - Jenkins, Edgar - Jenner, Philip N. - Karpenstein, Henry - Kupka, Edward - Lee, Hector - Leighly, John - Leland, Agnes C. - Loeb, Edwin M. - Loomis, Grant - Lovett, Hobart M. - McCrady, A. R. - McKenzie, Gordon - McWilliams, James M. Malkiel, Yakov - Markrich, Max - Meyer, Theodore R. - Michaelis, A. F. - Morley, S. G. - Roberts, John S. - Saroyan, William - Sledd, James - Starr, W. A. - Stevenson, Noel C. - Stewart, Mrs. Andrew W. - Stewart, George R. - Straubinger, O. Paul - Tamony, Peter Williams, Catherine Poey; California Academy of Science - University of California, Berkeley - Dept. of Geography, Univ. of Calif., Berkeley - University of California, Davis - University of California, Los Angeles - University of California, Riverside - California State Library Fresno State College - Henry E. Huntington Library - Los Angeles Public Library - Los Angeles State College - Oakland Public Library San Diego Public Library - San Francisco Public Library - San Jose Junior College - San Jose State College - Southwest Museum Library - Stanford University

Colorado (2-4). Davidson, Levette J. - Seely, Pauline A.; Colorado College - University of Colorado - Denver Public Library - University of Denver

Connecticut (5-3). Allen, Morse S. - Hughes, Arthur H. - Kökeritz, Helge - Minton, Arthur - Rounds, Stowell; Connecticut State Library - New Haven Public Library - Yale University

Delaware (2-0). Dunlap, A. R. - Weslager, C. A.

Florida (3-4). Hargrett, Lester - Pyles, Thomas - Reynolds, T. W.; Florida State Library - Florida State University - University of Florida - Miami Public Library

Georgia (1-1). Cheek, Ben F., Jr.; Atlanta Public Library Idaho (1-0). Morton, Maralyn 
Illinois (22-10). Collins, Charles E. - Droege, Geart - Edward, Edith F. - Franzen, Gösta - Fucilla, Joseph G. - Gelb, I. J. - Harris, Jesse W. - Hartman, A. A. - Johnson, E. Gustav - Jonikas, Petras - Kaganoff, Rabbi Benzion C. - McCutcheon, John T., Jr. - McQueen, Alexander - Manolatos, M. R. - Odell, Clarence B. - Pauls, John P. - Pollock, George H. - Sammons, Wheeler, Jr. - Smith, Elsdon C. - Steiner, Mrs. Mary Florence - Vest, Eugene B. - Yust, Walter; American Peoples Encyclopaedia - Bradley University - Chicago Public Library - University of Chicago - University of Illinois - The Newberry Library Northern Illinois State Teachers College - Northwestern University Rand McNally and Co. - Southern Illinois University

Indiana (4-3). Dean, Orpha M. - Kellogg, Allen B. - Orrick, Allan H. - Speer, David G.; Indiana State Library - University of Notre Dame - Purdue University

Iowa (0-2). State Historical Society - State University of Iowa

Kansas (0-2). Fort Hays Kansas State College - University of Kansas Kentucky (3-2). Clagett, Marjorie - Field, Thomas P. - Geiger, Mrs. Davis E.; University of Kentucky - Louisville Public Library

Louisiana (0-2). Louisiana State University - Southwestern Louisiana Institute

Maine (1-1). Chadbourne, Ava H.; Bowdoin College Library

Maryland (8-4). Cooley, Franklin D. - Geary, James A. - Gilbert, William H. - Hall, Richard N. - Malone, Kemp - Mutziger, John G. Shannon, George Pope - Vartanoff, Michael S.; Enoch Pratt Free Library - The Johns Hopkins University - University of Maryland U.S. Navy Hydrographic Office

Massachusetts (8-4). Clark, Donald T. - Duckert, Audrey R. Hough, Henry Beetle - McMullen, E. Wallace - Mag, Elsie - Starck, Taylor - Wilson, A. H. - Woolf, Henry B.; American Antiquarian Society - Boston Public Library - Harvard University - Peabody Museum, Salem

Michigan (11-6). Bickford, Roy M. - Foster, Theodore G. - Kushmar, Aneale L. - McCartney, Eugene S. - Marckwardt, Albert - Pulgram, Ernst - Roberts, R. D. - Seaman, William M. - Walton, Ivan H. - Winburne, John N. - Zelinsky, Wilbur; Detroit Public Library University of Detroit - Michigan Historical Commission - Michigan State College - University of Michigan - Wayne State University

Minnesota (4-2). Allen, Harold B. - Brainerd, E. L. - Brown, Samuel L. - Manfred, Frederick; Minneapolis Public Library - University of Minnesota

Missouri (4-4). Cochran, R. T. - McDermott, John Francis - Sealock, Richard B. - Zimmer, Gertrude M.; Kansas City Public Library - Missouri State Library - University of Missouri - St. Louis Public Library 
Montana (1-1). Ashton, William E.; Historical Society of Montana Nebraska (4-1). Ackerman, Louise M. - Gaffney, Wilbur G. - Meredith, Mamie - Pound, Louise; Nebraska State Historical Society

Nevada (2-1). Kramer, Fritz L. - Woods, H. J., Jr.; University of Nevada

New Hampshire (2-1). Kenny, Hamill - Stefansson, Vilhjalmur; Dartmouth College

New Jersey (10-2). Ames, Karl - D'Amato, Orlando - Eckerson, S. J. - Furnas, J. C. - Kinnaird, Clark - Philhower, Charles A. - Pugh, Mrs. H. - Talbot, Ashley F. - Umansky, Harlan L. - Williams, Margery M.; Demarest Memorial Library - Newark Public Library

New Mexico (4-1). Carlson, Helen - Duncan, R. M. - Jackson, John B. - Pearce, T. M.; University of New Mexico

New York (25-13). Abramson, Samuel H. - Abt, Henry E. (Brand Names Foundation) - Barnhart, Clarence L. - Barnhart, Robert K. Bijur, George - Brecher, Felix - Bryant, Margaret M. - Cross, Ephraim - Crowell, Thomas L. - Davis, Moshe - Giffin, Mary E. - Grauer, Ben - Hotchner, Cecilia A. - Irving, Walter V. - Jagendorf, Moritz - Maas, Ernest - Nunez, Benjamin - Read, Allen Walker - Rosenblum, Morris - Sheldon, Esther K. - Sonkin, Robert - Stein, Jess - Vicary, James M. - Wasson, R. Gordon - Wheeler, Helen P.; American Geographical Society - Brooklyn Public Library - University of Buffalo Cornell University - Funk and Wagnalls Co. - Grosvenor Library, Buffalo - National Audubon Society - The New York Historical Society New York Public Library - New York University - Pratt Institute Library - Queens Borough Public Library - Rochester Public Library North Carolina (4-4). McAtee, W. L. - MacClamroch, James J. W. - Royster, Hubert A. - Stoudemire, Sterling A.; Duke University Library - Gaston County Public Library - University of North Carolina - Womens College, Univ. of North Carolina

North Dakota (4-1). Beck, Richard - Caldwell, Robert A. - Georgacas, Demetrius J. - Pfaller, Benedict, OSB; University of North Dakota

Ohio (9-11). Alatis, James E. - Handshin, D. H. - Harder, Kelsie B. - Kenyon, John S. - Overman, William D. - Puckett, Newbell N. Raeth, C. J. - Raup, H. F. - Utley, Francis, L.; Bowling Green State University - Cincinnati Public Library - Cleveland Public Library John Carroll University - Kent State University - Miami University Ohio State University - Toledo Public Library - University of ToledoWestern Reserve University - The World Publishing Co.

Oklahoma (1-4). Criswell, E. H.; Oklahoma A. and M. College - Oklahoma City Libraries - University of Oklahoma - University of Tulsa

Oregon (1-2). Kraft, Walter C.; University of Oregon - Library Association of Portland 
Pennsylvania (10-9). Baker, Donald Gay - Baum, S. V. - Cohen, Hennig - Corcoran, Joseph N. - Haney, John Louis - Landreth, Symington P. - Mesko, Louis - Rogers, P. Burwell - Senn, Alfred Wolfeschlegelsteinhausenbergerdorff, Hubert Blaine, Sr.; Bryn Mawr College - Bucknell University - Lehigh University - Lincoln Library, Shippensburg - Pennsylvania Historical Society Ponnsylvania State Library - Pennsylvania State University - University of Pennsylvania - University of Pittsburg

Rhode Island (0-1). Brown University

South Carolina (2-2). Bradley, F. W. - Neuffer, Claude Henry; The Erskine College Library - University of South Carolina

South Dakota (1-1). Ehrensperger, Edward C.; University of South Dakota

Tennessee (1-3). Schemel, Margaret C.; Joint University Libraries Tennessee State Library and Archives - University of Tennessee

Texas (6-8). Atwood, E. Bagby - Cofer, D. B. - Dabbs, Jack A. Findley, Nell Green - Hunt, Ocie - Starnes, D. T.; Baylor University - Dallas Public Library - The Rice Institute - A. and M. College of Texas - Texas Christian University - University of Texas - Texas State College for Women - Texas Technological College

Utah (2-4). Hilbig, Frederick W. - Streadbeck, Arval L.; Brigham Young University - The Genealogical Society - Utah State Agricultural College - University of Utah

Vermont $(0-1)$. Vermont Historical Society

Virginia (5-4). Abbott, Charles C. - Gilliam, Charles Edgar - Hench, Atcheson L. - Percy, Alfred - Swem, E. G.; Norfolk Public Library Richmond Public Library - Virginia Historical Society - Virginia State Library

Washington (4-5). Hitchman, Robert - Perrin P. G. - Relander, Click - Waskey, Frank H.; Seattle Public Library - Spokane Public Library - State College, Pullman - Tacoma Public Library - University of Washington

West Virginia (5-2). Atha, Mrs. F. O. - Bush, Mrs. A. Kyle - Chrisman, Lewis H. - Mockler, William E. - Norona, Delf; West Virginia University - West Virginia Wesleyan College

Wisconsin (5-3). Cassidy, Frederic G. - Erlandson, Mrs. Willis J. Hanley, Louise - Hawley, Lee F. - Taube, Edward; Milwaukee Public Library - Wisconsin State Historical Society - University of Wisconsin

Wyoming (0-1). University of Wyoming

District of Columbia (7-6). Belden, Allen - Burrill, Meredith F. Haykin, David Judson - Heck, Louis - Kovaliv, Panteleimon K. Lyman, John - Wraight, A. J.; National Geographic Society - Smithsonian Institution - U.S. Army Map Service - U.S. Coast and Geodetic 
Survey - U.S. Dept. of the Interior, Office of Geography - U.S. Geological Survey

Alaska (1-0). DeArmond, Robert N.

Hawaii (1-0). Bryan, Edwin $H$.

Puerto Rico (0-1). University of Puerto Rico

Philippines (0-1). Manuel L. Quezon Institute

Canada (5-6). Bereskin, A. I. - Dupré, Gilles - G. M. Munroe (Board on Geographical Names) - Rudnychkyj, J. B. - Tepperman, I. J.; University of British Columbia - University of Manitoba - National Museum of Canada Library - Geographical Branch, Ottawa - Provincial Library, Victoria

Mexico (1-0). Tibón, Gutierre

Guatemala (1-0). Stahl, Gustavo

Belgium (2-2). Carnoy, A. - H. J. Van De Wijer; Office International de Librairie, Brussels - Bibliotheque de l'Université Louvain

Denmark (0-1). P. Haase and Son

England (2-2). King, Joyce - Wrenn, Charles W.; Bodleian Library - H. K. Lewis and Co., Ltd.

France (0-2). Librairie des Méridiens - Bibliothèque de l'Université, Paris

Germany (1-4). Krahe, Hans; Niedersächsische u. Universitätsbibliothek, Göttingen - Universitätsbibliothek, München - Freie Universität, Berlin - Gedenkbibliothek, Berlin

Spain (0-1). Facultad de Filosofia, Salamanca

Sweden (0-4). Gumperts Aktie Bolag - Humanistiska Biblioteket, Stockholms Högskola - Kungl. Biblioteket, Stockholm - A. B. Lundequistska Bokhandeln

South Africa (0-2). Public Library, Johannesburg - University of the Witwatersrand

Japan (2-0). Jinbo, Kiichi - Nishizawa, Hidemasa

Saudi Arabia (1-0). Rentz, George

U.S.S.R. (0-2). Bibliotheka Akademii Nauk, Leningrad - Bibliotheka Akademii Nauk, Moscow 